\title{
Influencia del tipo de feedback utilizado en el aprendizaje de una tarea motriz de equilibrio Influence of the type of feedback on balance motor tasks learning
}

\author{
Adrià Marco Ahulló, Xavier García Massó, Carlos García Osa, Isaac Estevan Torres
}

Universidad de Valencia (España)

\begin{abstract}
Resumen. El feedback que aportan los docentes de Educación Física puede favorecer el aprendizaje de los estudiantes en tareas motrices. La importancia de conocer la influencia del tipo de feedback a utilizar parece clave para programar con éxito las tareas a realizar. Sin embargo, actualmente la literatura existente no es capaz de advertir qué tipo de feedback es el más efectivo para el aprendizaje de una tarea motriz en adolescentes. El objetivo del presente estudio fue analizar la influencia de tres tipos de feedback terminal-verbal: i) sobre el resultado, ii) sobre el procedimiento o iii) sobre ambos (procedimiento-resultado) en el aprendizaje de una tarea motriz. La muestra estuvo compuesta por 30 alumnos/as de primer ciclo de la Educación Secundaria Obligatoria con edades comprendidas entre los 13 y 14 años (divididos aleatoriamente en los 3 grupos). Las mediciones de los datos se realizaron durante el pretest, postest y prueba de retención. Los resultados mostraron que los adolescentes que recibieron feedback sobre el procedimiento, obtuvieron unas puntuaciones más bajas en Raíz Media Cuadrática $(p<, 05)$, lo que se relaciona con un mayor rendimiento en la tarea, que quienes lo recibieron sobre el resultado o ambos. Los hallazgos obtenidos sugieren que, para favorecer el aprendizaje de una tarea motriz de equilibrio, se recomienda que el profesorado de Educación Física aporte feedback terminal-verbal sobre el procedimiento.
\end{abstract}

Palabras Clave: educación física, aprendizaje motor, equilibrio, retroalimentación, adolescencia.

\begin{abstract}
Depending on the type of feedback provided by physical education teachers, students' motor learning might be enhanced or not. The importance of understanding the influence of types of feedback seems to be essential to organize any motor learning process appropriately. Currently, it is not clear which type of feedback is the most effective for adolescents' motor learning. The purpose of this study was to examine students' balance motor learning according to the type of terminal-verbal feedback provided (i.e., feedback on result, feedback on process, or both). Thirty secondary school students aged between 13 and 14 years old (randomly distributed into 3 groups) participated in the study. Data collection was carried out using a balance motor task involving postural control on an unstable Wii Balance platform. Measurements were conducted at pretest, posttest, and follow-up. The results showed that students who received the terminal-verbal feedback on the process presented lower Root Mean Squared $(p<, 05)$, considered as a better performance, than those who received feedback on result or both types of feedback. Accordingly, to favor students' balance motor learning it is recommended that physical education teachers provide terminal-verbal feedback on process.
\end{abstract}

Key Words: physical education, motor learning, balance, feedback, adolescence.

\section{Introducción}

Durante los últimos años el sistema educativo español ha sufrido diversas variaciones para intentar adaptarse a las necesidades de una sociedad en constante cambio. La Educación Física no ha sido una excepción, y por ello, a lo largo de este proceso de evolución y cambio educativo se han ido planteando diferentes retos o desafíos que superar desde esta asignatura (e.g., promoción de la actividad física fuera del contexto escolar y de actitudes saludables, la educación en valores, etc.). López-Pastor, Brunicardi, Arribas, y Aguado (2016) conciben que, el desarrollo físico-motriz, la creación y recreación de la cultura física y la aportación al desarrollo integral del alumnado son los principales objetivos a alcanzar desde las aulas de Educación Física en la actualidad. La consecución del primer objetivo puede ayudar en gran medida a la superación de algunos de los retos que se plantean para la Educación Física actual, ya que, según el modelo propuesto por Stodden et al. (2008), se sugiere que altos niveles de competencia motriz se corresponderán con altos niveles de actividad física y a la inversa.

Ruiz Heredia, Lara Sánchez, López Gallego, Cachón Zagalaz y Valdivia Moral (2018) destacan que un cuarto del tiempo dedicado a las clases de Educación Física es considerado como no útil, y únicamente la mitad del tiempo total

Fecha recepción: 10-12-18. Fecha de aceptación: 11-05-19

Isaac Estevan Torres

isaac.estevan@uv.es de las sesiones se puede considerar como tiempo de compromiso motor. Si a todo esto le sumamos el reducido tiempo que se otorga a la asignatura de Educación Física en la Educación Secundaria Obligatoria en España en el currículum actual (véase Real Decreto 126/2014) y otros factores como la ratio profesor-alumnado, los docentes de esta materia deben utilizar recursos y métodos que favorezcan un proceso de enseñanza-aprendizaje eficaz durante el escaso tiempo del que se dispone con el alumnado para que adquieran y mejoren lo máximo posible sus habilidades motrices.

El proceso de enseñanza-aprendizaje se caracteriza por la interacción de un conjunto de variables referentes a la tarea a aprender. La práctica por sí sola resulta insuficiente para consolidar el aprendizaje; ya que es necesario un cierto conocimiento sobre el grado de aproximación al objetivo para contribuir a la reducción del error que los alumnos obtienen al realizar la tarea por primera vez (Fernández, 2006). En este punto, la labor docente resulta crucial y, por ello, la adquisición de competencias comunicativas es uno de los retos importantes que se plantean al profesorado de Educación Física ya que son fundamentales para la eficacia de los sistemas formativos y el aprendizaje del alumnado (Gallego-Ortega \& Rodríguez-Fuentes, 2018).

Una de las principales herramientas para favorecer el aprendizaje de tareas motrices es el feedback o retroalimentación, es decir, la información suplementaria que se le otorga al alumnado que realiza la tarea sobre su desempeño en esta. Los tipos de feedback se pueden clasificar según la procedencia del estímulo (intrínseco o extrínseco), la vía sen- 
sorial utilizada para captarlo (visual, auditivo o háptico), el momento en el que se proporciona (concurrente o terminal) y la información que se suministre [sobre el resultado (i.e., información relacionada con el resultado de la acción) o sobre el procedimiento (i.e., información relacionada con las características específicas de la composición del movimiento)] (Fujii, Lulic \& Chen, 2016; Keogh \& Hume, 2012; Magill, 1998; Schmidt, 1991; Sigrist, Rauter, Riener \& Wolf, 2013).

Aunque el tipo de feedback más estudiado en la literatura científica es el visual, el que con mayor frecuencia se ofrece durante las clases de Educación Física es el feedback verbal. El feedback verbal es considerado una subcategoría dentro del feedback auditivo, pudiendo diferenciar entre las señales sonoras (e.g., pitidos con el silbato) o directrices proporcionadas por un observador externo, que puede ser experto (e.g., el profesor de educación física) o no experto (e.g., un compañero de clase). La eficacia del feedback verbal viene supeditada al nivel de conocimiento que tiene el observador externo (e.g., docente) sobre la tarea encomendada (e.g., habilidad motriz). En este sentido, si la retroalimentación proporcionada es consistente y apropiada, el feedback verbal puede ser una herramienta efectiva para el aprendizaje motriz (Sigrist et al., 2013; van Vliet \& Wulf, 2006). Al respecto, pequeños cambios en la información verbal puede tener un efecto significativo sobre la forma de realizar las habilidades motrices propuestas y, consecuentemente, en el aprendizaje (Palmer, Matsuyama, Irwin, Porter \& Robinson, 2017).

Estudios centrados en comparar la eficacia de diferentes tipos de feedback se han realizado mayoritariamente en personas adultas, siendo significativamente menor el número de artículos publicados teniendo por protagonistas a niños y/o adolescentes (Sullivan, Kantak \& Burtner, 2008). En el ámbito de la actividad física y el deporte, los estudios en jóvenes han destacado la importancia del feedback verbal, frente a otros como el visual, mostrando que cuando se ofrecen varios tipos de feedback incluyendo el verbal (y aunque este sea erróneo), el aprendizaje se asocia a las características del feedback verbal aportado (Sigrist et al., 2013; van Vliet \& Wulf, 2006). Sin embargo, la aportación de feedback visual ha sido la más utilizada para la mejora de diferentes habilidades motrices como el golpeo (Weeks \& Kordus, 1998), el lanzamiento (Aiken, Fairbrother \& Post, 2012) y/o el control postural (McNevin, Shea, \& Wulf, 2003) entre otras. En este sentido, es importante analizar si cualquier tipo de feedback verbal favorece el aprendizaje de habilidades motrices o, si por el contrario, su eficacia depende de otros aspectos particulares como si la retroalimentación se centra en el resultado de la tarea, el procedimiento seguido o ambos por igual.

Los estudios centrados en analizar la eficacia de proporcionar información verbal sobre el conocimiento del resultado o sobre el procedimiento en diferentes tareas (Sharma, Chevidikunnan, Khan \& Gaowgzeh, 2016; Tzetzis, Kioumourtzoglou \& Mavromatis, 1997) no ofrecen hallazgos claros, sobre todo en población joven. De estos estudios se desprende que el feedback verbal de procedimiento parece ser más eficaz que el de resultado, sin embargo, estos estudios no incluyen retroalimentación en la que se combinen ambos tipos de feedback, de resultado y de procedimiento. Por ello, el objetivo del presente estudio fue analizar la influencia de tres tipos de feedback terminal-verbal: i) sobre el resultado, ii) sobre el procedimiento o iii) sobre ambos (procedimiento-resultado) en el aprendizaje de una tarea motriz de control postural. La hipótesis planteada fue que, en el proceso de aprendizaje motriz relacionado con una tarea de equilibrio, la retroalimentación terminal-verbal con una orientación al conocimiento del proceso es más eficaz que la orientación de conocimiento de resultado.

\section{Material y Método}

\section{Participantes}

El presente estudio cuasi-experimental estuvo compuesto por un total de 30 participantes (15 niños y 15 niñas) con edades comprendidas entre los 13 y 14 años $(M=13,28 ; D . T$. $=, 68)$, de una talla media de $1,64 \mathrm{~m}(D . T .=8,72)$ y un peso medio de 54,27 kg (D.T. =11,36). Los participantes no presentaban ningún tipo de alteración física o neurológica, y eran estudiantes de primer ciclo de secundaria. Los padres/madres y/o tutores de los adolescentes, firmaron un consentimiento informado sobre la participación en el estudio. Además, el Comité Ético de la Universidad de Valencia aprobó la realización del estudio.

\section{Procedimiento}

Los participantes fueron asignados aleatoriamente a uno de los tres grupos experimentales manteniendo un mismo tamaño muestral (10 participantes por grupo) donde a cada grupo se le proporcionó un tipo de feedback: Grupo 1, feedback terminal-verbal orientado al resultado; grupo 2, feedback terminal-verbal orientado al procedimiento y al grupo 3 se le proporcionó feedback terminal-verbal orientado al resultado y al procedimiento.

Antes de realizar la tarea por primera vez se informó a cada participante del objetivo de la misma, esto es mantener la plataforma (tabla de Freeman) lo más horizontal posible en cada intento, durante los 30 segundos. Las tareas se realizaron con los pies descalzos, manteniéndolos paralelos y separados una anchura igual a la de los hombros.

Cada participante realizó las pruebas de forma individual en una sala acompañado/a únicamente por un investigador, con conocimiento y experiencia en Educación Física y tareas de equilibrio, para reducir así cualquier tipo de interferencia en el desempeño de la tarea. Todos los participantes realizaron un pre-test y un post-test en la misma sesión en la que se realizó la práctica, y, además, 24 horas después realizaron una prueba de retención.

Durante las repeticiones pre-test, post-test y retención, el/la participante disponía de una marca situada en la pared a la altura de sus ojos que servía de punto de referencia. La distancia entre la pared y la zona en la que se colocó la plataforma fue de dos metros.

\section{Instrumentos}

Para la realización y mediciones de la tarea motriz de equilibrio se utilizó: a) una plataforma inestable de madera (60 x 40 $\mathrm{cm})$ con un eje central que permitiera rotación únicamente en el eje medio-lateral (tabla de Freeman). b) Encima de la tabla de Freeman se colocó una Wii Balance Board para recoger los datos durante las diferentes pruebas (pre-test, práctica, 
post-test y retención). Este instrumento ha sido validado como método de medición de centro de presiones tanto en adultos (Clark et al., 2010) como en jóvenes (Larsen, Jørgensen, Junge, Juul-Kristensen \& Wedderkopp, 2014).

\section{Protocolo}

Durante la primera sesión (cuya duración fue de 40 minutos aproximadamente) los participantes realizaron 21 repeticiones de la tarea motriz de 30 segundos de duración cada una (tres repeticiones pre-test, 15 repeticiones de práctica y tres repeticiones post-test), con un minuto de descanso entre repeticiones. En la segunda sesión se realizaron únicamente tres repeticiones de 30 segundos a modo de retención.

Las tres primeras repeticiones de la primera sesión permitieron conocer las condiciones iniciales de competencia en la tarea (pre-test), las 15 repeticiones siguientes se realizaron para practicar la tarea. Tras cada intento de la práctica, dependiendo del grupo al que pertenecía el/la participante, se le proporcionó retroalimentación sobre i) el resultado final de la tarea (feedback de resultado), ii) cómo había realizado la misma (feedback de procedimiento) o iii) ambos tipos de feedback. Por último, los tres intentos que se realizaron para concluir la primera sesión, permitieron conocer la adaptación de la competencia en la tarea al finalizar la intervención (posttest). La retención se realizó 24 horas después y consistió en la realización de tres repeticiones en las mismas condiciones que los pre y post-test con el objetivo de poder conocer si el aprendizaje motriz se mantuvo en el tiempo. La prueba se iniciaba unos segundos después de subirse a la plataforma y después de dar la señal verbal de inicio (preparado/a, listo/ a, ya).

Todos los grupos recibieron algún tipo de feedback terminal-verbal tras cada una de las 15 repeticiones realizadas como práctica de la tarea. El feedback se proporcionó una vez los sujetos habían bajado de la plataforma inestable. El feedback sobre el resultado consistió en informar del valor medio (en forma numérica) de la desviación de la plataforma con respecto a la horizontal, considerada con valor 0 . Cuanto más pequeña era la media de la desviación (cerca del 0), más se aproximaba al mantenimiento de la tabla lo más horizontal posible. El feedback sobre el procedimiento se caracterizaba por ofrecer indicaciones sobre diferentes estrategias que debían seguirse para realizar la tarea correctamente (Gandía, García-Massó, Marco-Ahulló \& Estevan, 2019). Estas indicaciones se clasificaron en cinco categorías basadas en la literatura publicada al respecto (Condon \& Cremin, 2014; Gandía et al., 2019). La primera categoría se corresponde con premisas relacionadas con la atención de la mirada durante la tarea; la segunda categoría corresponde a estrategias de compensación de las extremidades superiores y hombros; la tercera se corresponde a estrategias de compensación de las extremidades inferiores y cadera; la cuarta categoría corresponde con la presión ejercida por los pies en relación al contacto con la plataforma y la última categoría hace referencia a la actitud de los participantes frente a la tarea a realizar. El tercer grupo recibió ambos tipos de feedback, caracterizado por ofrecer indicaciones sobre el procedimiento y el resultado, siguiendo las mismas pautas que en los dos grupos anteriores.
Durante las repeticiones en el pre-test, post-test y retención, la competencia del alumnado en la tarea motriz de equilibrio se valoró por medio de los desplazamientos del centro de presiones en dirección medio-lateral. Además, cabe destacar que los sujetos no realizaron ninguna repetición de la tarea previamente al pre-test y que para evaluar el rendimiento en la tarea se realizó la media de los valores obtenidos en las tres repeticiones tanto en el pre-test, post-test y retención.

\section{Análisis de los datos}

Los datos captados por la Wii Balance fueron filtrados digitalmente de forma inicial con un filtro Butterworth de pasa-baja con una frecuencia de corte de $12 \mathrm{~Hz}$. La competencia motriz del alumnado se valoró calculando la Raíz Media Cuadrática (RMS por sus siglas en inglés). La RMS representa la distancia media del centro de presiones con respecto al punto 0 , por tanto, cuanto mayor sea el valor de la RMS menor competencia motriz en la tarea. Estudios previos en los que se valoró la competencia motriz en tareas de equilibrio, analizaron la RMS ya que ofrece información sobre la desviación con respecto al punto 0 (tabla totalmente en horizontal) que ha tenido lugar durante la tarea (e.g., MarcoAhulló et al., 2019). Por tanto, a mayor desviación con respecto a la horizontal, mayor será el valor de la RMS, lo que se interpreta como una menor competencia en la tarea.

\section{Análisis estadístico}

Todos los análisis estadísticos se realizaron con el paquete SPSS v.22 (IBM, Armonk, USA). En primer lugar, se comprobó el supuesto de normalidad en las variables dependientes mediante las pruebas de Kolmogorov-Smirnov. Tras comprobar que las variables cumplieron dicho supuesto, se utilizaron estadísticos descriptivos (media y desviación típica). A continuación, se aplicó un análisis múltiple de la varianza (MANOVA) de modelo mixto [grupo (3) x momento de medición (3)] para comprobar el efecto del tipo de feedback aplicado sobre la RMS. El seguimiento de los contrastes multivariados se realizó mediante contrastes univariados. Finalmente, se solicitaron comparaciones por pares con el ajuste de Bonferroni cuando fue necesario. El nivel de significación se fijó en $p=.05$. El tamaño del efecto de las diferencias se analizó con el cálculo de la eta parcial al cuadrado $\left(\eta_{\mathrm{p}}^{2}\right)$. Los valores de la $\eta_{\mathrm{p}}{ }^{2}$ son interpretados de la siguiente manera: $\eta_{\mathrm{p}}{ }^{2}<.01$ indica efectos aleatorios, entre .01 y .06 efectos pequeños, entre .06 y .14 efectos medios y $\eta_{\mathrm{p}}^{2}>.14$ efectos grandes (Cohen, 1988).

\section{Resultados}

Los contrastes mostraron un efecto principal del momento de la medición sobre la RMS $\left(\mathrm{F}_{(2,54)}=5.10 ; p<.009 ; c_{\mathrm{p}}{ }^{2}\right.$ $=.16)$. Sin embargo, no se encontró un efecto significativo del grupo $\left(\mathrm{F}_{(2,27)}=.50 ; p=.60 ; c_{\mathrm{p}}{ }^{2}=.04\right)$ ni de la interacción grupo $x$ momento de medición $\left(\mathrm{F}_{(4,54)}=1.80 ; p=.14 ; c_{\mathrm{p}}{ }^{2}=.12\right)$ en dicha variable. Las comparaciones por pares aparecen representadas en la Figura 1.

\section{Discusión}

El propósito de este estudio fue investigar si la aporta- 


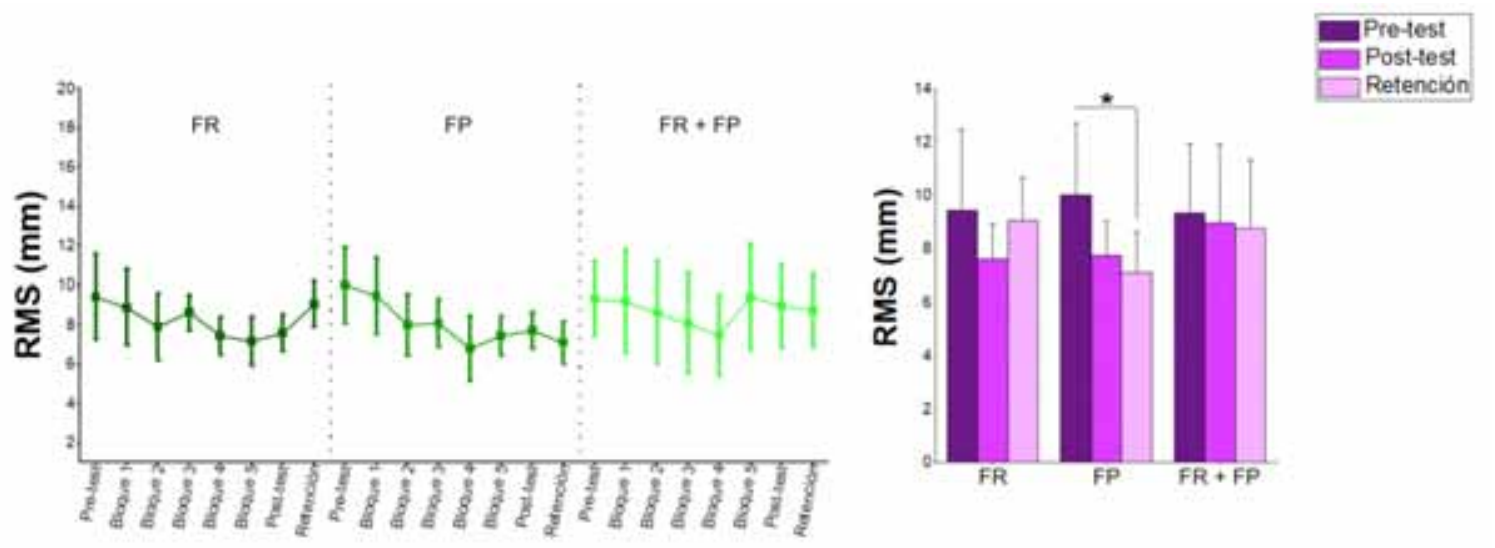

Figura 1. Comparaciones por pares de los tres grupos en pre-test, post-test y retención. Los gráficos de líneas de la izquierda muestran los valores medios cada tres repeticiones para cada grupo. Los gráficos de barras de la derecha muestran los valores medios de las tres repeticiones en el pre-test, post-test y retención para cada grupo. FR se refiere al grupo de feedback sobre el resultado, FP se refiere a el grupo de feedback sobre el procedimiento y FR+FP se refiere a al grupo que recibe ambos tipos de feedback. * $p<, 05$.

ción de feedback terminal-verbal orientado al resultado, al procedimiento o a ambos de forma conjunta podían influir en el aprendizaje de una tarea de equilibrio en estudiantes de Educación Física en el primer ciclo de Educación Secundaria. Los resultados del presente estudio indican que aportar feedback durante la realización de la tarea de equilibrio favorece el aprendizaje de la misma. Hallazgos similares han sido encontrados recientemente por Marco-Ahulló et al. (2019), quienes analizaron el efecto del uso de diferentes frecuencias de feedback concurrente-visual sobre el conocimiento del proceso en una tarea postural similar a la utilizada en el presente estudio. Los autores hallaron que era mejor utilizar este tipo de feedback en cualquiera de las dos frecuencias estudiadas (67\% y 100\%) que no hacerlo, lo cual refuerza la idea de que el feedback es capaz de incrementar el aprendizaje motriz de los estudiantes (Camacho Lazarraga, 2018; Vernetta \& López-Bedoya, 2010).

Nuestro estudio se diferencia del anteriormente citado (Marco-Ahulló et al., 2019) por dos elementos del tipo de feedback según cuándo y cómo se aporta. En primer lugar (cuándo), se ha elegido el feedback terminal por el hecho de que según la literatura es el más adecuado para el aprendizaje de tareas no complejas (i.e., sin muchos grados de libertad, realizada en un ambiente de laboratorio y que se pueda aprender en un único día) (Sülzenbrück \& Heuer, 2011). En segundo lugar (cómo), se ha utilizado el feeback verbal en lugar del feedback visual, lo cual se podría traducir en una mayor validez externa ya que parece ser que es una forma de proporcionar feedback que se lleva o que se puede llevar a cabo en cualquier aula de Educación Física sin mayor instrumental que la interacción docente-alumno/a. Prueba de ello es que el feedback verbal de procedimiento es uno de los tipos de feedback más utilizados en la instrucción deportiva (Hebert \& Landin, 1994).

Además, los resultados del presente estudio parecen confirmar la hipótesis inicialmente planteada respecto a que a los participantes que se les proporcionó retroalimentación terminal-verbal con una orientación al conocimiento del proceso obtuvieron mejores resultados que los participantes que recibieron retroalimentación terminal orientada al resultado o ambas. Es decir, nuestros resultados muestran diferencias significativas entre el pre-test y la retención en el grupo al que se le proporcionó únicamente retroalimentación orientada al proceso. No obstante, cabe señalar que en el grupo al que se le proporcionó ambos tipos de feedback también se advierten valores bajos en la variable RMS en la retención con respecto a los valores de los pre-test (aunque no significativos). Al respecto, un estudio previo que compara el uso del feedback de procedimiento frente al de resultado en una tarea de lanzamiento en una muestra de jóvenes adultos encontró resultados muy similares a los del presente estudio (Sharma et al., 2016). El uso de la información sobre el procedimiento consiguió crear mayores mejoras de los participantes en la tarea motriz frente a los que recibieron feeback sobre el resultado.

Nuestro estudio puede parecer contradecir lo propuesto por Tzetzis et al. (1997), quienes exponen que, frente al procedimiento, el conocimiento de los resultados junto con el establecimiento de metas es un método más efectivo para el aprendizaje de habilidades relacionadas con el baloncesto en niños. Estos mismos autores llegaron incluso a indicar que esta manera de proveer el conocimiento de los resultados era mejor que la conjunción de ambos conocimientos (resultados y procedimiento). Sin embargo, como advierten Locke y Latham (1985), el efecto positivo del feedback puede no ser únicamente debido a éste sino a que el mismo se complementa con el establecimiento de metas, aumentando su efecto sobre el aprendizaje. Cabe señalar que en nuestro estudio no se incluyó el establecimiento de metas como un factor de retroalimentación. Por ello, futuros estudios deberían abordar el análisis no sólo del efecto del tipo de feedback sino el de ambos factores, el establecimiento de metas y el tipo de feedack sobre el aprendizaje de tareas de equilibrio.

Pese a pretender controlar diferentes factores que pudieran influir en los resultados, como el factor edad y bagaje motriz, nuestro estudio no está exento de limitaciones. A lo largo del manuscrito se ha insistido en la necesidad de estudiar la aplicación de los diferentes tipos de feedback y de las diferentes formas posibles de hacerlo en poblaciones de niños y adolescentes. Una de las razones por las que se ha hecho hincapié en adolescentes es que tienen un menor bagaje motriz que las poblaciones adultas o adultos jóvenes por, entre otros aspectos, el hecho de que parecen haber 
tenido menos tiempo para experimentar con su cuerpo y que este está continuamente cambiando (Gallahue, Ozmun \& Goodway, 2012). La variable del bagaje motriz es importante ya que quizás cuando se tiene una experiencia previa en las diferentes tareas no es tan relevante que se aprenda la tarea, sino «reaprender» y/o modificar la técnica anteriormente adquirida, pudiendo ser diferente el tipo de feedback necesario para el aprendizaje de una tarea que para la modificación de una conducta o «reaprendizaje». El reaprendizaje ha sido estudiado recientemente por Krause (2017) quién intentó modificar la técnica de inicio y de giro en nadadores expertos utilizando el conocimiento del procedimiento o la combinación del conocimiento del procedimiento y del resultado. Los resultados extraídos de dicho estudio parecen revelar que el uso de los dos tipos de feedback de forma conjunta eran la mejor opción para el reaprendizaje o corrección de la técnica utilizada.

El presente estudio se ha centrado en el uso del feedback verbal por el hecho de que es uno de los tipos de retroalimentación más utilizados en la instrucción deportiva y el cuál creemos que en este momento va a ser más sencillo de llevar a la práctica en las diferentes clases de Educación Física. De acuerdo a la rápida evolución que experimenta la tecnología y los dispositivos electrónicos, se debería investigar si la utilización de dispositivos electrónicos de bajo coste como elemento proveedor de feedback (de resultado, de procedimiento o ambos) utilizando esta tecnología en un ambiente más ecológico puede favorecer el aprendizaje de las tareas motrices en Educación Física.

\section{Conclusiones}

El hallazgo principal de este estudio ha sido que, aunque el aprendizaje de una tarea motriz se ve beneficiado tras la aportación de cualquiera de los feedback estudiados en este trabajo, la retroalimentación terminal-verbal enfocada al procedimiento en adolescentes resulta ser la que parece producir un mayor aprendizaje motriz. Además, frente a otros tipos de feedback, el terminal-verbal orientado al procedimiento es el que parece retener el aprendizaje durante más tiempo.

En consecuencia, cabe destacar que los y las docentes de Educación Física deben de tener en cuenta las palabras o frases a utilizar para proporcionar las instrucciones a la hora de facilitar la mejora de las habilidades de estabilidad de los adolescentes. Según el tipo de información ofrecida, esta puede tener cierto impacto sobre la realización y aprendizaje de la tarea por parte del alumnado.

\section{Agradecimientos}

Los autores quieren agradecer a los centros educativos, los docentes de Educación Física, así como al alumnado la participación voluntaria en este estudio.

\section{Referencias}

Aiken, C. A., Fairbrother, J. T., \& Post, P. G (2012). The Effects of Self-Controlled Video Feedback on the Learning of the Basketball Set Shot. Frontiers in Psychology, 3. https:// doi.org/10.3389/fpsyg.2012.00338
Camacho Lazarraga, P. (2018). Efecto del foco atencional sobre el aprendizaje de las habilidades deportivas individuales. Retos: Nuevas Tendencias en Educación Física, Deportes y Recreación, 36, 451-457. Retrieved https:// recyt.fecyt.es/index.php/retos/article/view/64428/41405

Clark, R. A., Bryant, A. L., Pua, Y., McCrory, P., Bennell, K., \& Hunt, M. (2010). Validity and reliability of the Nintendo Wii Balance Board for assessment of standing balance. Gait \& Posture, 31(3), 307-310. https://doi.org/10.1016/ j.gaitpost.2009.11.012

Condon, C., \& Cremin, K. (2014). Static balance norms in children. Physiotherapy Research International: The Journal for Researchers and Clinicians in Physical Therapy, 19(1), 1-7. https://doi.org/10.1002/pri.1549

Fernández, J. P. (2006). Efectos de la información de retorno sobre el resultado en el aprendizaje de tareas motoras. Revista Iberoamericana de Educación, 37(5), 1-4.

Fujii, S., Lulic, T., \& Chen, J. L. (2016). More Feedback Is Better than Less: Learning a Novel Upper Limb Joint Coordination Pattern with Augmented Auditory Feedback. Frontiers in Neuroscience, 10. https://doi.org/ 10.3389/fnins.2016.00251

Gallego-Ortega, J. L., \& Rodríguez-Fuentes, A. (2018). Percepciones del profesorado sobre competencias comunicativas de futuros maestros de educación física. Revista Internacional de Medicina y Ciencias de la Actividad Física y del Deporte, 18(71), 479-492. https:// doi.org/10.15366/rimcafd2018.71.005

Gandía, J., García-Massó, X., Marco-Ahulló, A., \& Estevan, I. (2019). Adolescents’ Postural Control Learning According to the Frequency of Knowledge of Process. Journal of Motor Learning and Development, 1-11. https://doi.org/10.1123/jmld.2017-0042

Gallahue, D. L., Ozmun, J. C., \& Goodway, J. D. (2012). Understanding Motor Development: Infants, Children, Adolescents, Adults (Ed. 7). New York(NY): McGraw-Hill Education.

Hebert, E. P., \& Landin, D. (1994). Effects of a learning model and augmented feedback on tennis skill acquisition. Research Quarterly for Exercise and Sport, 65(3), 250257. https://doi.org/10.1080/02701367.1994.10607626

Keogh, J. W. L., \& Hume, P. A. (2012). Evidence for biomechanics and motor learning research improving golf performance. Sports Biomechanics, 11(2), 288-309. https://doi.org/10.1080/14763141.2012.671354

Krause, D. (2017). Effects of additional knowledge of results on modifying highly practiced acyclic swimming techniques with knowledge of performance. International Journal of Sports Science \& Coaching, 12(6), 737-746. https://doi.org/10.1177/1747954117738894

Larsen, L. R., Jørgensen, M. G., Junge, T., Juul-Kristensen, B., \& Wedderkopp, N. (2014). Field assessment of balance in 10 to 14 year old children, reproducibility and validity of the Nintendo Wii board. BMC Pediatrics, 14, 144. https://doi.org/10.1186/1471-2431-14-144

Locke, E. A., \& Latham, G. P. (1985). The Application of Goal Setting to Sports. Journal of Sport Psychology, 7(3), 205-222. https://doi.org/10.1123/jsp.7.3.205

Magill, R. A. (1998). Research Lecture: Knowledge is more than we can talk about: implicit learning in motor skill 
acquisition. Research Quarterly for Exercise and Sport, 69(2), 104-110. https://doi.org/10.1080/ 02701367.1998.10607676

Marco-Ahulló, A., Sánchez-Tormo, A., García-Pérez, J. A., Villarrasa-Sapiña, I., González, L. M., \& García-Massó, X. (2019). Effect of Concurrent Visual Feedback Frequency on Postural Control Learning in Adolescents. Journal of Motor Behavior, 51(2), 193-198. https://doi.org/10.1080/ 00222895.2018.1454397

McNevin, N. H., Shea, C. H., \& Wulf, G. (2003). Increasing the distance of an external focus of attention enhances learning. Psychological Research, 67(1), 22-29. https:// doi.org/10.1007/s00426-002-0093-6

Palmer, K. K., Matsuyama, A. L., Irwin, J. M., Porter, J. M., \& Robinson, L. E. (2017). The effect of attentional focus cues on object control performance in elementary children. Physical Education and Sport Pedagogy, 22(6), 580588. https://doi.org/10.1080/17408989.2017.1294667

López-Pastor, V. M., Brunicardi, D. P., Arribas, J. C. M., \& Aguado, R. M. (2016). Los retos de la Educación Física en el siglo XXI. Retos: Nuevas Tendencias en Educación Física, Deporte y Recreación, 29, 182-187.

Ruiz Heredia, C. M., Lara Sánchez, A. J., López Gallego, F. J., Cachón Zagalaz, J., \& Valdivia Moral, P. (2018). Análisis del tiempo de clase en EF y propuestas para su optimización. Retos: Nuevas Tendencias en Educación Física, Deportes y Recreación, 35, 126-129. Retrieved from https:/recyt.fecyt.es/index.php/retos/article/view/ 61880/40841

Schmidt, R. A. (1991). Frequent Augmented Feedback Can Degrade Learning: Evidence and Interpretations. In J. Requin \& G. E. Stelmach (Eds.), Tutorials in Motor Neuroscience (pp. 59-75). https://doi.org/10.1007/97894-011-3626-6_6

Sharma, D. A., Chevidikunnan, M. F., Khan, F. R., \& Gaowgzeh, R. A. (2016). Effectiveness of knowledge of result and knowledge of performance in the learning of a skilled motor activity by healthy young adults. Journal of Physical Therapy Science, 28(5), 1482-1486. https:// doi.org/10.1589/jpts.28.1482

Sigrist, R., Rauter, G, Riener, R., \& Wolf, P. (2013). Augmented visual, auditory, haptic, and multimodal feedback in motor learning: a review. Psychonomic Bulletin \& Review, 20(1), 21-53. https://doi.org/10.3758/s13423-012-0333-8

Stodden, D. F., Goodway, J. D., Langendorfer, S. J., Roberton, M.A., Rudisill, M. E., Garcia, C., \& Garcia, L. E. (2008). A Developmental Perspective on the Role of Motor Skill Competence in Physical Activity: An Emergent Relationship. Quest, 60(2), 290-306.

Sullivan, K. J., Kantak, S. S., \& Burtner, P. A. (2008). Motor learning in children: feedback effects on skill acquisition. Physical Therapy, 88(6), 720-732. https://doi.org/ 10.2522/ptj.20070196

Sülzenbrück, S., \& Heuer, H. (2011). Type of visual feedback during practice influences the precision of the acquired internal model of a complex visuo-motor transformation. Ergonomics, 54(1), 34-46. https://doi.org/10.1080/ 00140139.2010 .535023

Tzetzis, G., Kioumourtzoglou, E., \& Mavromatis, G. (1997). Goal Setting and Feedback for the Development of Instructional Strategies. Perceptual and Motor Skills, 84(3_suppl), 1411-1427. https://doi.org/10.2466/ pms.1997.84.3c.1411

van Vliet, P. M., \& Wulf, G. (2006). Extrinsic feedback for motor learning after stroke: What is the evidence? Disability and Rehabilitation, 28(13-14), 831-840. https://doi.org/10.1080/09638280500534937

Vernetta, M., \& López-Bedoya, J. (2010). Análisis de diferentes categorías del feedback en dos formas organizativas del medio gimnástico. European Journal of Human Movement, 4(0), 113-129.

Weeks, D. L., \& Kordus, R. N. (1998). Relative frequency of knowledge of performance and motor skill learning. Research Quarterly for Exercise and Sport, 69(3), 224230. https://doi.org/10.1080/02701367.1998.10607689
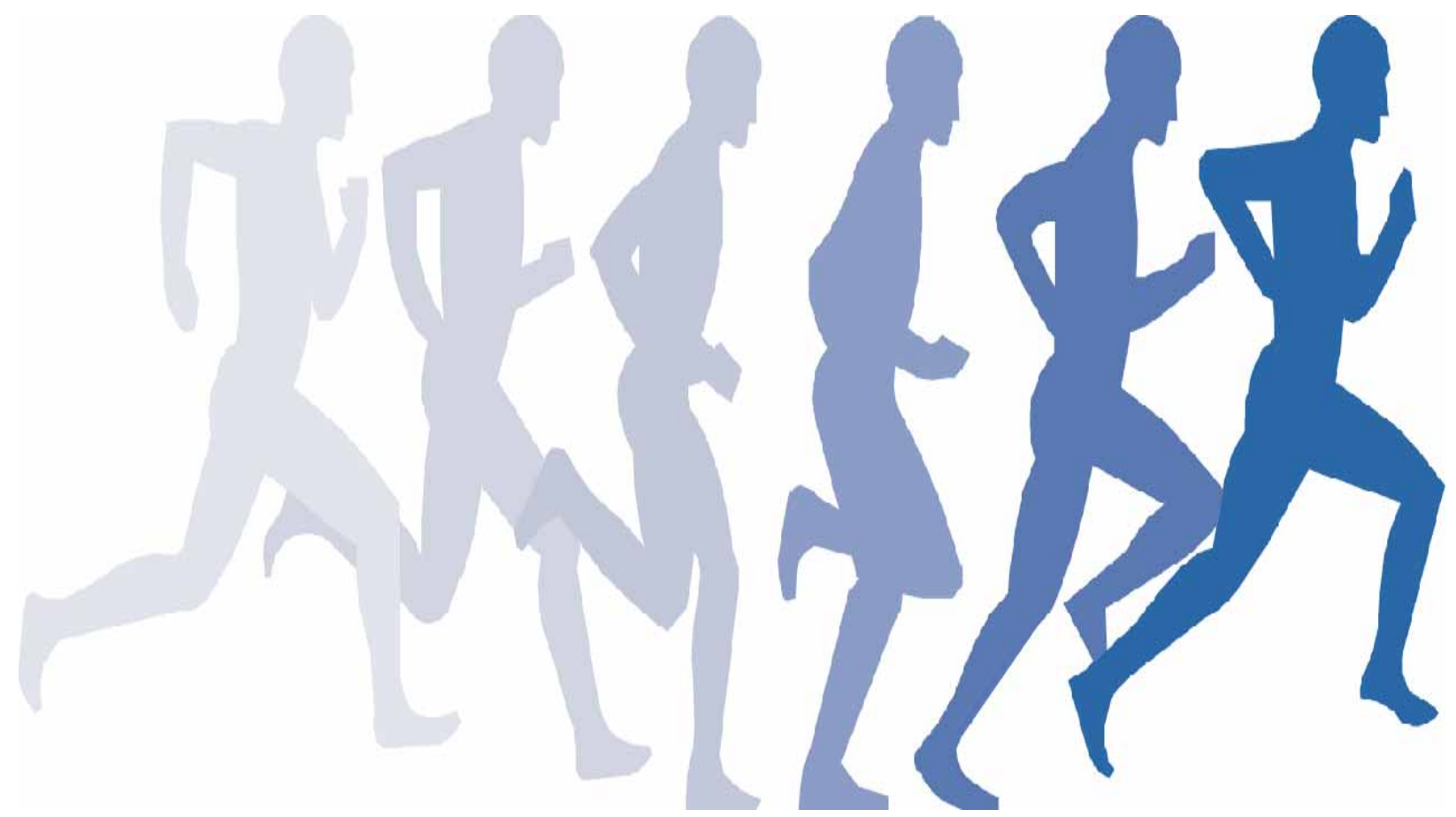